Exemplaria Classica

Journal of Classical Philology

22, 2018, 13-29

ISSN 1699-3225

\title{
ARS ADEO LATET ARTE SUA : AUTOUR DE L'ÉLÉGIE CRYPTOLOGIQUE 3.14 DES TRISTES D'OVIDE ET D'HYGIN, PRÉFET DE LA BIBLIOTHÈQUE PALATINE
}

\author{
GaUtHIER Liberman \\ Université Bordeaux-Montaigne et \\ École Pratique des Hautes Etudes \\ gauthier.liberman@orange.fr
}

\section{SUMMARY}

We try to corroborate Rudolf Merkel's idea that Ovid's friend, the philologist and prefect of the Palatine Library Hyginus is actually the addressee of the last poem of book $\mathbf{3}$ of the Tristia collection. In order to do so, before identifying the cryptological signa laid by Ovid, we discuss the position and personality of Hyginus, the position of Pompeius Macer, who was also busy with the Palatine Library. We also endeavour to explain passages of various authors (Augustus, Ovid, Suetonius, Tzetzes on Callimachus), suggest emendations of some of these passages and discuss various issues pertaining to the achievement of ancient librarians and to ancient literary cryptology.

\section{KEY WORDS}

Ovid; Hyginus; Macer; Callimachus; Augustus; cryptology; library and librarians.

\section{RÉSUMÉ}

On essaie de corroborer l'hypothèse de Rudolf Merkel selon laquelle l'affranchi d'Auguste, philologue, ami d'Ovide et préfet de la Bibliothèque Palatine Hygin est bien le destinataire de la dernière pièce du livre 3 des Tristes. Pour ce faire, avant d'identifier les signa cryptologiques disposés par Ovide, on évoque les fonctions et la personnalité d'Hygin ainsi que les fonctions de Pompeius Macer, qui s'est aussi occupé de la Bibliothèque Palatine. Au cours de la discussion on interprète plusieurs passages d'auteurs divers (Auguste, Ovide, Suétone, Tzetzès sur Callimaque), on suggère des émendations de certains de ces passages et l'on évoque aussi des questions relatives au travail des bibliothécaires de l'Antiquité et à la cryptologie littéraire antique.

\section{MOTS CLEFS}

Ovide; Tristes; Hygin; Macer; Callimaque; Auguste; cryptologie; bibliothèque et bibliothécaires.

Fecha de recepción: 21/05/2018

Fecha de aceptación y versión final: 2/10/2018 


\section{Georgii Luckii piae memoriae}

L'helléniste et latiniste Rudolf Merkel, cité, avec Ézéchiel Spanheim et Theodor Birt, par A. E. Housman ${ }^{1}$ pour illustrer le cas des philologues chez qui l'érudition parvient presque à compenser l'absence de "faculté critique ", formula, dans une édition mémorable des Tristes parue en $1837^{2}$, l'idée géniale que le correspondant auquel, dans le quinzième ${ }^{3}$ et dernier poème du livre 3 , Ovide demande de faire une place notamment à ce livre ${ }^{4}$, n'est autre que le célèbre disciple d'Alexandre Polyhistor, lui-même polymathe, polygraphe et, selon un renseignement que fournit Suétone (de gramm. 20.2 p. 443 Kaster), ami très proche d'Ovide et préfet de la Bibliothèque Palatine, C(aius) Iulius Aug(usti) 1(ibertus) Hyginus ${ }^{5}$, l'Hygin sous le nom prestigieux duquel furent

${ }^{1}$ A.E. Housman, M. Manilii astronomicon liber primus, Londres 1903, XLV. Ce jugement doit être relativisé: comparé, par exemple, aux éditeurs d'Ovide et en particulier des poèmes d'exil dans la Collection des Universités de France, Merkel est le plus distingué des critiques. Les prolégomènes (près de trois cent pages) de son édition critique des Fastes (Berlin 1841) ne sont certainement pas moins érudits qu'intelligents, mais ils ne sont peut-être pas non plus moins intelligents qu'érudits.

${ }^{2}$ R. Merkel, P. Ovidii Nasonis Tristium libri quinque et Ibis, Berlin 1837, 217. Merkel (âgé de 26 ans en 1837!) est peut-être, dans une certaine mesure, tributaire, pour son idée, de l'hypothèse ancienne, qu'il réfute (p. 388-90), reconnaissant, derrière Ibis, dans le poème de ce nom, Hygin (ainsi encore J. Carcopino, Rencontres de l'histoire et de la littérature romaines, Paris 1963, 147). F. Bömer, P. Ovidius Naso, Die Fasten, Heidelberg 1957, I, 24 qualifie de "fraglich » l'idée (cf., entre autres, P. van de Woestijne, «Un ami d'Ovide, C. Julius Hyginus», Musée Belge 33, 1929, 31-45) que trist. 1.7 a pour destinataire Hygin: c'est, à mon avis, une hypothèse dépourvue du moindre fondement.

${ }^{3}$ La numérotation 1-14 provient de la fusion des pièces $4 \mathrm{a}$ et $4 \mathrm{~b}$ dans la plupart des manuscrits. Merkel, P. Ovidii Nasonis Tristium, 166 défend cette fusion. Elle est encore dans sa recension de 1851 (Teubner) mais disparaît dans la révision du volume concerné de l'Ouidius Merkelianus par R. Ehwald en 1884 (Teubner) et n'est plus en vogue aujourd'hui. Sur le problème de la séparation des poèmes, voir généralement S.J. Heyworth, «Dividing Poems», in O. Pecere et M.D. Reeve, edd., Formative Stages of Classical Traditions: Latin Texts from Antiquity to the Renaissance, Spoleto 1995, 117-48 et, à propos des Tristes, F. Ursini, "Questioni di unitarietà nei Tristia di Ovidio", Maia 67, 2015, 354-83; il justifie (367-70) la division de 3.4. en deux élégies et remarque (381-2) que le nombre résultant d'élégies (15) pour le livre 3 est caractéristique de l'architectonique ovidienne (sur ce point, voir aussi G. Liberman, «La critique du texte des Hérö̈des», in L. Rivero, $\mathrm{M}^{a} \mathrm{C}$. Álvarez, Rosa $\mathrm{M}^{a}$ Iglesias, J. A. Estévez, edd., Viuam! Estudios sobre la obra de Ovidio - Studies on Ovid's poetry, Huelva Classical Monographs 10, Huelva 2018, 78).

${ }^{4}$ Voir 3.14.25-6 Hoc quoque nescioquid nostris adpone libellis, Diuerso missum quod tibi ab orbe uenit, avec S.G. Owen, Ovid, Tristia Book III, Oxford 18932, 73 et G. Luck, P. Ovidius Naso. Tristia, herausgegeben, übersetzt und erklärt, Heidelberg 1977, II, 229.

${ }^{5}$ Voir, sur Hygin, outre les travaux mentionnés dans la suite, R.A. Kaster, C. Suetonius Tranquillus, De grammaticis et rhetoribus, Edited with a Translation, Introduction, and Commentary, Oxford 1995, 205-14 et B.M. Levick, T.J. Cornell, «C. Julius Hyginus», in T.J. Cornell, éd., The Fragments of the Roman Historians, Oxford 2013, I, 475-81. Si nous avons raison de penser être en mesure de montrer que l'élégie d'Ovide a pour destinataire $\mathrm{Hy}-$ gin, Kaster et Levick-Cornell ont tort de croire qu'Ovide s'adresse à un successeur d'Hygin à la tête de la Bibliothèque Palatine. 
rangées des œuvres apocryphes ${ }^{6}$. Personne n'était mieux placé pour réaliser les vœux d'Ovide que l'administrateur des bibliothèques (la grecque et la latine) du temple d'Apollon Palatin ${ }^{7}$. En même temps, Ovide devait éviter ou (la distinction importe) paraître éviter le risque de causer du tort ${ }^{8}$ à un tel correspondant - préfet d'une bibliothèque publique, certes, mais impériale, utilisant des esclaves de la maison du prince, et non, comme la bibliothèque $\mathrm{du}$ Portique d'Octavie, " urbaine $»^{9}-$ en le désignant autrement que d'une manière obscure et pour ainsi dire cryptologique. L'objet premier de la présente note est de corroborer l'idée de Merkel en montrant qu'Ovide, qui ne nomme explicitement ni Hygin ni la fonction au titre de laquelle il sadresse à lui, fournit au lecteur très attentif les moyens ${ }^{10}$ d'identifier le destinataire de la lettre et sa fonction : le poème contient en effet des indices plus nombreux que ceux vus par Merkel lui-même ou par Peter ${ }^{11}$ dans son célèbre recueil des fragments des historiens romains. Si nous parvenons à nos fins, nous serons de surcroît, ce semble, fondés à faire valoir l'allusion cachée au nom et à la

${ }^{6}$ Voir Peter, Historicorum Romanorum reliquiae, Volumen alterum, Leipzig 1906, CI : de C. Iulio Hygino liberto Augusti, quocum illi (qui libros de astrologia et genealogias siue fabulas scripserunt uel scripsisse dicuntur) nil nisi Hygini nomen commune habent (...). Aujourd'hui on ne prend pas toujours la peine de distinguer clairement du véritable Hygin les autres; il est vrai que certains attribuent au préfet de la Palatine les œuvres que Peter et d'autres lui enlevaient (voir Kaster, De grammaticis, 207-8 et Levick-Cornell, «C. Julius Hyginus», 475-6).

${ }^{7}$ Sur cette bibliothèque et le complexe où elle s'insère, voir le mémoire fouillé de M. Corbier, «De la maison d'Hortensius à la curia sur le Palatin», MEFRA 104, 1992, 871-916 (version contractée chez M. Corbier, Donner à voir, donner à lire. Mémoire et communication dans la Rome ancienne, Paris 2006, 163-79); V.M. Strocka, «Römische Bibliotheken», Gymnasium, 88, 1981, 307-9 et Casson, Libraries, passim. J'utilise le terme " préfet » en m'autorisant de la terminologie vaticane toujours en vigueur Bibliothecae Apostolicae Vaticanae praefectus.

${ }^{8}$ Voir Carcopino, Rencontres, 85 et R. Syme, History in Ovid, Oxford 1978, 39 et 767. Syme suggère dubitativement que le destinataire du poème qui nous intéresse est Brutus, " who was charged with Ex Ponto I-III ". " The Tristia, remarque-t-il, carry no names, save one poem to an accomplished lady called Perilla (III.7)». E. Smith est l'auteur d'une thèse de l'Université de Pennsylvanie intitulée " Names and Naming in Ovid's Exile Poetry » (2012). Je n'en connais que le résumé, où, à propos des Tristes, l'auteur dit ceci: " Attempts to find "real identities" of the poems' addressees are shown to be largely pointless; those identities are left deliberately open, to be determined by readers of the poems ".

${ }^{9}$ Voir T. Mommsen, Römisches Staatsrecht, Berlin, $1887^{3}$, I, 330 n. 1; V. Gardthausen, Augustus und seine Zeit, Leipzig 1891, I, 965 et II, 580; M. Ihm, «Die Bibliotheken im alten Rom», Centralblatt für Bibliothekswesen, 12, 1893, 526; O. Hirschfeld, Die kaiserlichen Verwaltungsbeamten bis auf Diocletian, Zweite neubearbeitete Auflage, Berlin 1905, 304. Confusion chez L. Casson, Libraries 2001, 97-8.

${ }^{10}$ Voir 1.5a.7 positis pro nomine signis avec Syme, History in Ovid, 76. L'édition des Tristes à laquelle je renvoie, non sans signaler les passages controversés, est celle de J.B. Hall, Ovidius, Tristia, Stuttgart/Leipzig 1995. Il y a peut-être une double entente en 3.14.45-46, dicere saepe aliquid conanti (turpe fateri) Verba mihi desunt dedicique loqui. Ovide feint de croire que l'exil lui a ôté la facilité de parole (c'est un topos chez lui): à ce sens premier s'ajoute peut-être l'intention de laisser entendre qu'il est des noms qu'il ne peut dire.

${ }^{11}$ Peter, Historicorum Romanorum reliquiae, Volumen alterum, CIII. 
fonction d'Hygin contre la thèse selon laquelle l' " exil " d'Ovide est une fiction littéraire. Comment, en effet, serait-il plausible qu'un exilé purement littéraire recourût à la cryptologie pour désigner, comme le destinataire d'une requête personnelle ayant pour objet la publicatio (mise à disposition du public) d'œuvres de lui, un personnage officiel contemporain doté sinon de l'intention, du moins de la possibilité matérielle d'y satisfaire? Mais rien n'est trop improbable pour satisfaire le désir de défendre une thèse paradoxale, surtout quand elle repose sur la " littérarisation ", la " fictionalisation » d'un élément qui passe pour avoir un ancrage historico-biographique ${ }^{12}$. S'il ne faut pas prendre Ovide au pied de la lettre et croire à l'historicité de tout ce qu'il raconte à son lecteur, néanmoins la mise en scène littéraire de son " exil» nous en verrons quelques aspects étonnants - n'implique pas le caractère fictif de ce dernier. La reconnaissance des signa disposés par Ovide permettra non seulement de mieux comprendre la dernière pièce du livre 3 des Tristes mais aussi de mieux en établir le texte. Avant de procéder à cette reconnaissance, il est indispensable d'évoquer quelques questions relatives à Hygin et à un autre érudit, Pompeius Macer, qui s'est occupé de la Bibliothèque Palatine et que certains identifient au Macer très lié à Ovide, destinataire de am. 2.18, de pont. 2.10 et mentionné dans ce dernier recueil en $4.16 .6^{13}$.

Les mots qu'utilisent Suétone (de gramm. 20.2) pour désigner la charge d'Hygin qui nous intéresse, praefuit Palatinae Bibliothecae, se recoupent avec la terminologie grecque ${ }^{14}$ et avec l'expression qu'emploie Ovide lui-même dans le premier poème du livre 3 des Tristes, custos praepositus (v. 67-8), passage où il est explicitement question du $\beta \imath \beta \lambda_{1} 0 \varphi v{ }^{\prime} \lambda \alpha \xi^{15}$ de la Bibliothèque Palatine. En ce custos J. Lipsius, De bibliothecis syntagma, Anvers $1607^{2}$, 22 (= T. Hendrickson, Ancient Libraries and Renaissance Humanism, The De Bibliothecis of Justus Lipsius, Boston 2017, 98) et, indépendamment, $\mathrm{O}$. Hirschfeld ${ }^{16}$ suggérèrent de reconnaître Hygin; dans un article sur

\footnotetext{
${ }^{12}$ Voir G. Liberman, «L'elogio pindarico di Teosseno rivisitato», in S. Caciagli, éd., Eros e genere in Grecia arcaica, Bologne 2017, 125-70, à propos de l'éloge pindarique de Théoxène. La critique post-moderne veut que l'éloge soit la commande d'un patron et que le poète n'expose pas un sentiment personnel. Cette interprétation fait (c'est ce que je crois montrer) abstraction du texte, sur lequel on plaque, d'une manière tout à fait caractéristique et contraire aux exigences de la philologie, une théorie générale toute faite en négligeant l'articulation du texte et sans se préoccuper aucunement d'une compréhension juste du détail.

${ }^{13}$ Le Macer de Tristes 4.10.44 est Aemilius Macer (voir Syme, History in Ovid, 179).

${ }^{14}$ Voir P. White, «'Pompeius Macer' and Ovid», CQ 42, 1992, 213 n. 9.

${ }^{15}$ Voir, sur ce mot, A. Langie, Les bibliothèques publiques dans l'ancienne Rome et dans l'Empire Romain, thèse Fribourg (Suisse) 1908, 145.

${ }_{16}$ O. Hirschfeld, Untersuchungen auf dem Gebiete der römischen Verwaltungsgeschichte, Erster Band, Die kaiserlichen Verwaltungsbeamten bis auf Diocletian, Berlin 1877,189 n. $5\left(=1905^{2}, 302\right.$ n. 6$)$.
} 
les bibliothèques de la Rome antique qui fit date, Max $\mathrm{Ihm}^{17}$ fut de ceux qui exprimèrent leur assentiment. À en croire Peter, Historicorum Romanorum reliquiae, Volumen alterum, CIII-CIV, Ovide, dans le dernier poème du livre 3 (vers 25-6), demande pour son livre à Hygin la faveur que ce dernier a refusée d'après la première pièce, où Ovide fait parler son livre en quête d'hospitalité dans la Ville : quaerentem (sc. fratres meos) frustra custos $<m>e$ sedibus illis Praepositus sancto iussit abire loco. L'invraisemblance d'un tel scénario saute aux yeux : le refus du custos dans la première pièce aurait dissuadé le poète de revenir à la charge en présentant la requête du poème final, qui ne fait aucune allusion à un premier refus. Peter s'est laissé égarer par l'illusion que l'ordre de succession des deux poèmes coïncide avec la chronologie des événements qu'ils sont censés évoquer ${ }^{18}$. Par la place qu'il assigne à ces poèmes, Ovide encadre le recueil -15 poèmes formés de deux groupes de sept séparés par l'unique poème dont Ovide nomme le destinataire, sa collègue la poétesse Perilla - de deux pièces dont la première paraît répondre à la dernière. En renversant le rapport entre ordre de succession livresque et ordre chronologique attendu, Ovide paraît placer très efficacement tout le recueil sous l'éclairage sombre de l'infortune et de l'échec, repulsa $(3.1 .81)^{19}$. Faut-il admettre que la pièce 3.14 constitue la requête réellement présentée à Hygin et qui se heurta à une fin de non-recevoir (3.1) ? C'est impossible, car alors 3.14.25-26 demanderaient l'hospitalité pour un livre, le livre 3, privé de sa première pièce : puisque celle-ci relate le refus opposé à la requête, elle ne saurait avoir été comprise dans le livre qui constitue l'objet de la requête. C'est la preuve que nous avons à faire à une mise en scène littéraire. En imaginant que les trois bibliothèques romaines, la Palatine (3.1.59-68), celle du Portique d'Octavie (69-70) et celle de l'Atrium Libertatis (71-2) ${ }^{20}$, refusent l'accès à son livre, persona non grata, Ovide semble chercher à apitoyer son lecteur, à émouvoir Hygin et peut-être à prévenir un refus réel en mettant en scène un refus imaginé. Relevons que dans la première pièce c'est le livre d'Ovide qui est censé parler, tandis que c'est Ovide qui parle dans la dernière pièce, où, si l'on admet qu'Hygin est visé, l'inflexible custos de 3.1.67 est présenté sous des traits plus individuels et plus aimables. En faisant parler son livre, Ovide paraît se distancier du refus qu'il met en scène. Selon Suétone (de gramm.

${ }^{17} \mathrm{Ihm}$, «Die Bibliotheken», 519 n. 32.

${ }^{18} \mathrm{G}$. Wartenberg, Quaestiones Ovidianae quibus agitur de Tristium Ibidis epistularumque quae ex Ponto inscribuntur temporibus, diss. Berlin 1884, 37 et Owen, Tristia, VIII avaient pourtant bien vu le " véritable » rapport chronologique entre la première et la dernière pièce du livre 3 .

${ }^{19}$ Pour le soin apporté par Ovide à l'architecture de ses recueils, voir par exemple, à propos des Pontiques, J. Irigoin, Le poète grec au travail, Paris 2009, 485-92. Je relève l'encadrement des livres 1-3 par les deux lettres adressées à Brutus, respectivement la première (prologue) et la dernière et trentième (épilogue): voir Syme, History in Ovid, 80. Les deux lettres présumées adressées à Hygin jouent le même rôle dans le livre 3 des Tristes.

${ }^{20}$ Voir Ihm, «Die Bibliotheken», 515-19. 
20.2), Hygin a fini sa vie dans une paupertas ${ }^{21}$ très grande - « a melancholy fate for one of the higher librarians, and perhaps the sequel to some scandal or action of political imprudence " (Syme, History in Ovid, 11222). Hygin, qui avait vraisemblablement entre 70 et 74 ans en 10 ap. J.-C. ${ }^{23}$, paya-t-il le fait de s'être montré complaisant envers son ami Ovide ${ }^{24}$ ? Kaster, De grammaticis, $212^{25}$ fonde sur le fait qu'Hygin a fini sa vie " in absolute poverty " l'idée que, déjà âgé en 10 ap. J.-C. (" c.70 years old "), il n'était plus « head of the library at that age " et que par conséquent il ne peut être le destinataire de trist. 3.14. À en croire Suétone (de gramm. 20.2) alléguant un propos de l'historicus consularis Caius Clodius Licinius ${ }^{26}$, Hygin était non seulement mort admodum pauperem mais, jusqu’à sa mort, avait été matériellement soutenu par le généreux consulaire, liberalitate sua quoad uixerit sustentatum. Si l'on poussait jusqu'au bout le raisonnement de Kaster, qui implique l'aisance matérielle du chef de la Bibliothèque Palatine ${ }^{27}$, Hygin ne pourrait pas même avoir été titulaire de ce poste, puisque, si les mots de Suétone ont un sens, Hy-

${ }^{21} \mathrm{Il}$ faut se méfier de ce mot, qui peut signifier non " dénuement » mais « modestie de ressources " (cf. R.G.M. Nisbet, N. Rudd, A Commentary on Horace, Odes, Book III, Oxford 2004, 23, 210 et 288).

22 Voir aussi Peter, Historicorum Romanorum reliquiae, Volumen alterum, CIV.

${ }^{23}$ Peter, Historicorum Romanorum reliquiae, Volumen alterum, CII fait naître entre 64 et 60 av. J.-C. celui que Suétone (de gramm. 20.1) présente comme a Caesare puerum Romam adductum Alexandria capta (47 av. J.-C.).

${ }^{24}$ Hypothèse déjà formulée par Woestijne, «Un ami d'Ovide» et A. Grisart, «La publication des Métamorphoses: une source des récits d'Ovide», Atti del Convegno Internazionale Ovidiano (Sulmona, maggio 1958), Rome 1958, 2, 125-55.

${ }^{25}$ Approuvé par Levick-Cornell, «C. Julius Hyginus», 475. $113)$.

${ }^{26}$ Consul dans la seconde moitié de 4 ap. J.-C. (cf., par exemple, Syme, History in Ovid,

${ }^{27}$ Peter, Historicorum Romanorum reliquiae, Volumen alterum, CIV croit aussi que les émoluments attachés par Auguste au poste d'Hygin étaient substantiels et il se réfère pour cela à Hirschfeld, Die kaiserlichen Verwaltungsbeamten, 302 ss., mais, en lisant ces pages, on découvre que Hirschfeld s'étonne de la faiblesse du traitement d'un procurator bibliothecarum (toutes les bibliothèques impériales, selon Hirschfeld) sous Antonin le Pieux, Lucius Baebius Aurelius Iuncinus ( "rangirte also in die unterste Gehaltsklasse der Procuratoren », observe Ihm, «Die Bibliotheken», 524): 60000 sesterces, « etwa 12550 Mark », selon Ihm (on trouve sur des sites en ligne qu’à la même époque le Chancelier du Reich est réputé recevoir 4000 marks mensuels et que sous les Antonins 60000 sesterces annuels équivalent à 20000 francs mensuels de 1986). Selon Hirschfeld et H.-G. Pflaum, Les carrières procuratoriennes équestres sous le Haut-Empire romain, Paris 1960, II, 679, la faible dotation s'explique parce que « les compétences de l'emploi de L. Baebius Aurelius Juncinus se bornaient à la partie administrative de la direction des bibliothèques ». À la suite de E. Bowie, «Libraries for the Cesars », dans J. König, A. Oikonomopoulou, G. Woolf, edd., Ancient Libraries, Cambridge 2013, 258, G.W. Houston, Inside Roman Libraries, Book Collections and Their Management in Antiquity, Chapel Hill 2014, 243 n. 108 doute, " given his youth and salary ", que Iuncinus ait été " procurator of all the libraries » tout en reconnaissant qu'il ne saurait avoir été chargé d'une seule. Pour illustrer la paupertas d'Hygin préfet de la Palatine, C.B. Bunte, De C. Julii Hygini, Augusti liberti, vita et scriptis, diss. Marburg 1846, 14 cite, d'après des sources littéraires, les cas de grammatici dans le besoin. 
gin avait " durant sa vie " (quoad uixerit) connu des difficultés financières, qui, évidemment, n'ont pu que s'aggraver en cas de disgrâce. Il me paraît en effet abusif et contraire aux mots de Suétone de limiter les difficultés d'Hygin à la période postérieure à sa sortie de charge présumée ${ }^{28}$, comme le fait Kaster: "Clodius' report - that H. ended his days in absolute poverty, relying for an extended period on Clodius' support - clearly implies that in his later days $\mathrm{H}$. was not still active on the Palatine, nor even deriving income from his teaching ». Au contraire, rien, me semble-t-il, n'empêche qu'Hygin soit resté en fonction jusqu'à un âge avancé, que Clodius ait suppléé à l'insuffisance de son traitement et qu'Hygin, après la cessation de son activité et peut-être sa disgrâce, soit mort non " in absolute poverty ", interprétation tendancieuse et peut-être fautive du latin, mais " hors de toute aisance ", voire " dans une véritable modestie de ressources ", car, nous l'avons signalé, paupertas est un " faux ami »; j'ajoute que, dans une séquence affirmative, admodum peut signifier " complètement » (cf. J. P. Krebs, J. H. Schmalz, Antibarbarus der lateinischen Sprache ${ }^{7}$, Bâle 1905, I, 98) mais aussi "vraiment ", " plutôt " (cf. l'OLD ${ }^{2}$ s. v. admodum et surtout l'étude exhaustive de O. Janssen, L'expressivité chez Salvien de Marseille. Étude sur l'usage de quelques particules dans le latin chrétien. Première partie. Les adverbes, thèse Nimègue 1937, 56-82). Kaster nous semble verser dans la contradiction en admettant simultanément le soutien libéral de Clodius et l'« absolute poverty " d'Hygin. La contradiction s'efface si l'on prête un sens plus mesuré à admodum pauperem. Nous croyons que l'identification, à laquelle nous procéderons, des signa permettant de reconnaître Hygin dans le destinataire de l'élégie consommera la réfutation de la thèse de Kaster. Owen, Tristia, 70-1 suggère qu'Hygin avait entrepris de superviser la publication sinon de l'ensemble des Tristes, du moins du livre 3 et que, comme, au début du poème, Ovide regrette que son livre ne puisse concevoir l'espoir de trouver place dans les bibliothèques publiques, de même, à la fin, il donne à Hygin des raisons de ne pas craindre de le mettre dans les rayons de sa bibliothèque privée. Le distique final, qualemcumque igitur uenia dignare libellum, Sortis et excusa conditione meae, ne se rapporte ni nécessairement ni, à mon avis, vraisemblablement à Hygin en tant que propriétaire d'une bibliothèque privée. De surcroît, quel sens peut-il y avoir à persuader de faire une place dans sa bibliothèque personnelle au livre d'Ovide un homme que l'on suppose s'être chargé de la publication de ce même livre ${ }^{29}$ ?

${ }^{28}$ Voir J. Christes, Sklaven und Freigelassene als Grammatiker und Philologen im antiken Rom, Wiesbaden 1979, 77: « die Bemerkung des Licinius scheint sich (...) auf einen längeren Zeitraum zu beziehen ".

${ }^{29}$ La suggestion d'Owen s'appuie sur la variante fautive conficis = colligis (v. 5). G. Luck, Tristia, herausgegeben, übersetzt und erklärt, Heidelberg 1967, I, et 1977, II, 227-8 adopte aussi conficis et veut que, selon une acception technique, ce verbe signifie " procurer une édition critique ». En admettant même que ce verbe ait un sens aussi précis, la question " Es-tu en train 
Dans le passage où il dit qu'Auguste avait demandé à Pompeius Macer de ne pas mettre à la disposition du public certains écrits de Jules César, Suétone (Vie de Jules César 56.7 p. 44 Kaster) rapporte que l'empereur avait confié à ce Pompeius Macer la charge d'organiser les bibliothèques, cui ordinandas ${ }^{30}$ bibliothecas delegauerat. Syme, History in Ovid, 73 est de ceux pour qui Macer était " chargé des bibliothèques de Rome " ${ }^{31}$, mais cette interprétation est très probablement erronée : les bibliothèques romaines ont été fondées à des dates diverses et il est a priori invraisemblable qu'elles aient été organisées par le même homme. Suétone doit avoir en vue l'organisation d'une bibliothèque particulière, en l'occurrence la bibliothèque grecque et la bibliothèque latine du temple d'Apollon Palatin, fondées le 9 octobre 28 av. J.-C. Ihm, «Die Bibliotheken», 517 n. 18 me paraît avoir raison de considérer

de procurer une édition critique, l'Art d'aimer mis à part, qui fut fatal à son auteur, de mes poèmes ? " me paraît sonner tout à fait faux dans le contexte. Hall, Ovidius, choisit la variante suscipis, " prends-tu fait et cause pour ", qui paraît cadrer mieux avec le contexte. Bunte, $D e C$. Julii Hygini, Woestijne, «Un ami d'Ovide» et Grisart, «La publication», sont d'avis qu'Hygin s'est chargé de l'édition des Métamorphoses. Je ne vois pas comment on peut raisonnablement concilier cette idée avec 3.14.19-24, où Ovide regrette que les Romains connaissent cette œuvre sous une forme non révisée, incorrectum opus! Selon le poète, s'il n'était déjà mort, c'est-à-dire s'il ne se trouvait dans la position d'un auteur dont l'œuvre est publiée après sa mort sans qu'il l'ait achevée et polie, elle aurait pu connaître une gloire plus sûre, certius a summa nomen habere manu (22). Ovide a-t-il plutôt écrit clarius, " une gloire plus radieuse "? Comparer met. 6.425 clarum uincendo nomen habebat. L'incorrectum opus d'Ovide ne manque pas de sel si l'on se souvient qu'Hygin a relevé chez Virgile quae inperfecta reliquisse poeta uel parum feliciter instituisse arguitur (O. Ribbeck, Prolegomena critica ad Vergilii opera maiora. Leipzig 1866, 119; nous indiquerons plus bas d'autres travaux sur Hygin critique de Virgile).

${ }^{30}$ Sur le sens d'ordinare, voir White, "Pompeius Macer'», 213 et Kaster, De grammaticis,

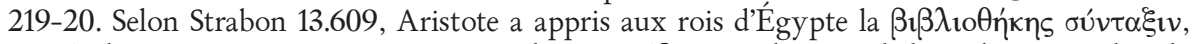
c'est-à-dire bibliothecae ordinationem. Il est stupéfiant que l'auteur de l'article ordino dans le $T L L$ 9.2.938.39 ss. n'ait pas cru bon de faire un sort au tour bibliothecas ordinare, qui apparait deux fois chez Suétone et chez lui seulement. On trouve les deux occurrences, mais sans explication, à l'article bibliotheca 2.1957.74 et 85. L'OLD s. u. ordino 3a entend « organiser ». Dans son exposé sur la pinacographie callimaquéenne, O. Schneider, Callimachea, Leipzig 1873, II, 298 réemploie l'expression de Suétone à propos du travail d'Alexandre d'Étole et de Lycophron (ut qui bibliothecam a Philadelpho conditam ordinauerint) et la description qu'il donne (p. 301-2) de ce travail est peut-être la meilleure exégèse de bibliothecas ordinare.

${ }^{31}$ Voir aussi R. Syme, The Augustan Aristocracy, Oxford 1986, 361: " Augustus appointed Macer to organize the libraries ". Bowie, "Libraries», 240-1 et 246-7 fonde là-dessus une construction au moins hasardeuse: après la nomination d'Hygin à la tête de la Palatine et de Melissus comme organisateur de la bibliothèque du Portique d'Octavie, Macer aurait été chargé d'une coordination " interbibliothécaire ". Christes, Sklaven, 75-6 soutenait une thèse analogue. F. Millar, Rome, the Greek World and the East, II, Government, Society and Culture in the Roman Empire, Chapel Hill 2004, 154 critique Pflaum, Les carrières, I, 12 pour avoir cru que le distingué Macer - qu'il soit procurator bibliothecarum, comme le veut Pflaum et le suppose Millar, ou que le passage de Suétone concernant Macer ne se rapporte qu’a la Palatine - devait occuper un poste plus élevé que l'affranchi Hygin. S. Demougin, Prosopographie des chevaliers romains julio-claudiens, Rome 1992, 109 parle aussi, à propos de Macer, de « la direction des bibliothèques, à Rome même ». Pflaum imagine que l'emploi confié à Macer était " une sorte de 'Ministère de l'information'»! 
cela comme prouvé - ou virtuellement prouvé - par le rapprochement de la notice où Suétone (de gramm. 21.3 p. 443 Kaster) enseigne qu'Auguste avait confié à l'affranchi de Mécène Caius Maecenas Melissus l'organisation des bibliothèques (la grecque et la latine) du Portique d'Octavie, fondées après la mort de Marcellus, intervenue en 23 av. J.-C.: quo (Auguste) delegante curam ordinandarum bibliothecarum in Octauiae porticu suscepit. Il est toutefois permis de se demander s'il n'y a pas une omission accidentelle dans le texte de Suétone relatif à Pompeius Macer : le parallèle du passage portant sur Melissus suggère, conformément à la phraséologie connue par les inscriptions et indiquée par Ihm, bibliothecas <Apollinis> ou <templi Apollinis ${ }^{32}$. La différence terminologique entre cette ordinatio bibliothecarum et le tour utilisé à propos d'Hygin, praefuit Palatinae Bibliothecae, amène White, "Pompeius Macer'», 214 à établir une distinction très rigide entre l'organisation et la direction d'une bibliothèque. S'il a raison, on ne peut plus dire, comme on le répète souvent depuis au moins E. Reusch, Dissertatio inauguralis de bibliothecis Romanorum, Helmstadt 1734, 22, que Macer fut le premier directeur de la Bibliothèque Palatine et Hygin le second: cette distinction très rigoureuse amène logiquement à professer, avec Peter, Historicorum Romanorum reliquiae, Volumen alterum, 111, qu'Hygin fut le premier directeur ${ }^{33}$, et ceci de 28 av. J.-C. jusqu'en 10 ap. J.-C. au moins, en admettant que trist. 3.1.67-68 se rapporte à Hygin, puisque la composition du livre 3 remonte au printemps et à l'été $10^{34}$. En réalité, comme le voulaient aussi Ihm, «Die Bibliotheken», 517 et Hirschfeld, Die kaiserlichen Verwaltungsbeamten, 302, il est tout à fait possible qu'Hygin soit le successeur (direct ou non) de Pompeius Macer à la tête des deux bibliothèques du temple d'Apollon. En effet, il serait compréhensible que le directeur d'une bibliothèque nouvellement créée procédât à son ordinatio, travail qui n'a plus à être accompli par la suite, du moins à la même échelle qu'au début. Alors, le

32 On pourrait rapprocher du texte transmis ou du moins d'un supplément tel que <suas $>$ CIL 6.2132 proc(urator) rat(ionum) summ(arum) priuatarum bibliothecarum Augusti si O. Gilbert, Geschichte und Topographie der Stadt Rom im Altertum, Dritte Abteilung, Leipzig 1890, 339 n. 3 avait raison de comprendre qu'il s'agit de la Bibliothèque Palatine, mais voir Ihm, «Die Bibliotheken», 524. L'absence, dans la phrase de Suétone relative à Macer, de l'équivalent de in Octauiae porticu dans celle sur Melissus suggère à Christes, Sklaven, 75 et Bowie, «Libraries», 247 que, s'agissant de Macer, bibliothecas s'entend de plusieurs bibliothèques romaines. Cela me paraît fourvoyé.

${ }_{33}$ Voir déjà S. Lürsenius, De templo et bibliotheca Apollinis Palatini liber singularis, Franeker 1719, 189 et Bunte, De C. Julii Hygini, 11. Houston, Inside Roman Libraries, $221 \mathrm{n}$. 14 reproche à juste titre à R. Fehrle, Das Bibliothekswesen im alten Rom: Voraussetzungen, Bedingungen, Anfänge, Fribourg en Brisgau 1986, 77-81 de penser qu'Hygin fut le premier à organiser la Bibliothèque Palatine. Selon Kaster, De grammaticis, 211, " Macer's work must have preceded the administration of $\mathrm{H}$., who was clearly head of the library some time after its formation ".

${ }^{34}$ Voir Syme, History in Ovid, 38. 
directeur d'une bibliothèque ne peut plus être avec autant d'à-propos désigné comme celui qui l'organise, ordinat : tel est, je le suggère, le cas d'Hygin, en opposition à Pompeius Macer et à Maecenas Melissus, dont la fonction de premier directeur inclurait celle, digne d'être mise en relief, d'organisateur. White, «'Pompeius Macer", 214 illustre la distinction romaine entre ordinator (pour parler comme Schneider, Callimachea, II, 301) et directeur d'une bibliothèque par la différence qui sépare le pinacographe Callimaque du bibliothécaire en chef Zénodote, mais la pertinence de la comparaison est des plus sujettes à caution. En effet, outre que, si je ne m'abuse, la période d'activité de Zénodote et celle de Callimaque ne coïncident pas ${ }^{35}$, l'ordinatio d'un Macer ou d'un Melissus ne saurait guère être sinon comparée, du moins commensurable au travail de pinacographie, novateur et à l'échelle des collections alexandrines, réalisé par Callimaque ${ }^{36}$ postérieurement à l'ordinatio accomplie par Zénodote, Alexandre d'Étole et Lycophron ${ }^{37}$. L'hypothèse

\footnotetext{
${ }^{35}$ Sur la chronologie callimaquéenne, voir L. Lehnus, Maasiana \& Callimachea, Milan 2016, 73-6 et 111-17.

${ }^{36}$ Voir R. Pfeiffer, Storia della filologia classica dalle origine alla fine dell'età ellenistica, Naples 1973 (original paru à Oxford 1968), 181-223; R. Blum, Kallimachos, The Alexandrian Library and the Origins of Bibliography, Translated from the German by H.H. Wellisch, Madison 1991 (original paru à Francfort 1977). Exemple significatif chez Irigoin, Histoire du texte de Pindare, Paris 1952, 33. Ordre de grandeur de la proportion des collections: 400000 rouleaux (bibliothèque du palais à Alexandrie à l'époque de Callimaque, voir V.M. Strocka, «Römische Bibliotheken», 302); « the library of the Forum of Trajan, quite likely Rome's largest, had space for but 10,000 in its Greek chamber. The Palatine Library, with half as many bookcases, held far fewer " (Casson, Libraries, 99 à propos de la section grecque des bibliothèques romaines; section grecque et romaine comprises, la bibliothèque trajane devait compter environ 20000 rouleaux d'après Casson, Libraries, 88). Nigel Wilson m'informe amicalement que le chiffre de 400000 rouleaux pour la bibliothèque du palais à Alexandrie est beaucoup trop élevé pour être plausible et que $R$. Bagnall suggère de le réduire à 40000 ("Alexandria: the Library of Dreams", Proceedings of the American Philosophical Society 146, 2002, 355-356). Je constate que W.A. Becker eut la même idée (Paulys Real-Encyclopädie, Zweite Auflage, I 2, Stuttgart 1866, 2374 n. 2).

${ }^{37}$ Voir R. Pfeiffer, Callimachus, Oxford 1949, II, 349 à Callimaque fr. 453; C. Wendel, Die griechisch-römische Buchbeschreibung verglichen mit der des vorderen Orients, Halle 1949, 69-75; Blum, Kallimachos, 226-30. Si j'ose utiliser ici le mot ordinatio, c'est que le mot grec diorthose, repris de Jean Tzetzès par Pfeiffer (cf. Test. 14b Pfeiffer), me paraît trop étroit si l'on ne voit dans le travail des trois érudits que l'emendatio. Sur le problème, voir par exemple F. Ritschl, Friderici Ritschelii opuscula philologica, Leipzig 1866, I, 138-40; H. Keil chez Ritschl, Friderici Ritschelii opuscula, 224-6; Schneider, Callimachea, II, 298-302 (enclin à élargir le sens de " diorthose »); H. Pusch, Quaestiones Zenodoteae, dans Dissertationes Hallenses, Halle 1890, XI, 119-216, 203-6 (défend le sens étroit); F. Susemihl, Geschichte der griechischen Litteratur in der Alexandrinerzeit, Leipzig 1891, I, 337 et 891-2 (Tzetzès a choisi un mot inadéquat); F. Schmidt, Die Pinakes des Kallimachos, Berlin 1922, 33-42 (dans la lignée de Schneider, Callimachea); Pfeiffer, Storia della filologia classica, 184 (défend le sens étroit) et Blum, Kallimachos, 104-23 (dans la lignée de Pfeiffer, mais avec plus de souplesse); F. Montanari, «Ekdosis. A Product of the Ancient Scholarship», dans F. Montanari, S. Matthaios et A. Rengakos, edd., Brill's Companion to Ancient Scholarship, Leiden 2015, II, 641-72, 642 (élargit démesurément le sens du mot, tractatio, pour le restreindre aussitôt à
} 
plausible d'un recours à des auxiliaires ${ }^{38}$ et d'une direction d'équipe ne change rien à l'énormité du labeur. Le complément apporté plus tard aux tables callimaquéennes par Aristophane de Byzance ${ }^{39}$, bibliothécaire en chef après Zénodote, Apollonios de Rhodes et Eratosthène ${ }^{40}$, s'inscrit dans le travail de Callimaque sans s'y substituer.

emendatio). Le célèbre scholium Plautinum (cf. Pfeiffer, Callimachus, II, XCVII Test. 14d et Pfeiffer, Storia della filologia classica, 175, 184, 213-14) paraît rendre le grec de Tzetzès

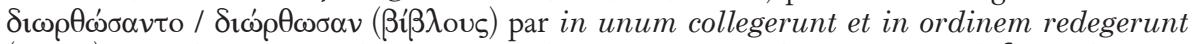
(libros), mais il se peut que l'humaniste italien se soit trouvé face à un texte différent du nôtre

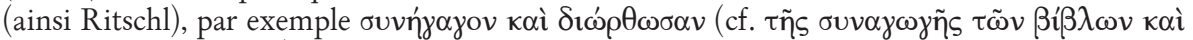
$\delta 10 \rho \theta \omega \dot{\sigma \varepsilon \omega s}$, Test. 14c) et qu'il ait mal compris $\delta i \omega \rho \rho \omega \sigma \alpha \nu$, lequel, signifiant parfois " remettre de l'ordre " (dans une province révoltée, par exemple), a pu être tiré du côté de "mettre en ordre ». Pour un exemple antique et certifié de rendu surprenant au moyen du mot grec qui nous occupe, comparer, à propos des exempla maiorum exolescentia, le rendu de reduxi par $\delta \omega \rho \theta \omega \sigma \alpha ́ \mu \eta \nu$ dans les res gestae diui Augusti 8 (cf. W. Weber, Princeps. Studien zur Geschichte des Augustus, Stuttgart/Berlin 1936, 170*). Si, avec le seul $\delta 1 \omega \rho \theta \omega \sigma \alpha v$, Tzetzès a employé un mot inadéquat, alors notre humaniste aura retrouvé le vrai sens, plus large! Pfeiffer a d'ailleurs tort de reprocher à cet humaniste, qui utilise l'expression in ordinem redigere, d'en méconnaître le sens, qui serait " ranger (des auteurs) dans le canon ", comme dans le passage célèbre de Quintilien inst. 1.4.4 (cf. 10.1.54). Le tour s'emploie en fait dans des sens divers, " disposer, ranger ", "faire rentrer dans le rang (quelqu'un ou quelque chose de récalcitrant) " (TLL IX.2.955,63-66 et 72, article qui omet le passage sus-mentionné de Quintilien et n'est pas mieux fait que celui relatif à ordinare).

${ }^{38}$ Voir U. von Wilamowitz, Hellenistische Dichtung in der Zeit des Kallimachos, Berlin 1924, I, 213.

${ }^{39}$ Voir A. Nauck, Aristophanis Byzantii grammatici Alexandrini, Halle 1848, 243-52; K. Nickau, "Aristophanes von Byzanz zu den pinakes des Kallimachos», RhM110, 1967, 34653; Pfeiffer, Storia della filologia classica, 221-2; A. Slater, Aristophanis Byzantii fragmenta, Berlin/New York 1986, 134-6 et 143.

${ }^{40}$ L'idée que Callimaque a été préfet de la bibliothèque (Blum, Kallimachos, passim; Casson, Libraries, 38-9) se heurte au témoignage de P. Oxy. 1241 col. II 1 (= Test. 13 Pfeiffer) et (voir F. Ritschl, Die Alexandrinischen Bibliotheken unter den ersten Ptolemäern (...) nach Anleitung eines platonischen Scholions, Breslau 1838, 19) ne repose que sur le scholium Plautinum, Callimacus aulicus regius bibliothecarius. Le grec de Tzetzès présumé

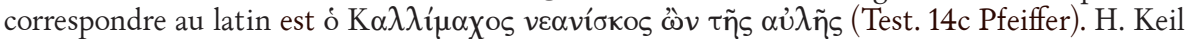

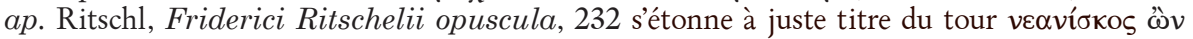
$\tau \tilde{\eta} \varsigma$ aủ入ñ , que Schneider, Callimachea, II, 299 tente de corriger en partant du présupposé que Callimaque fut bien préfet. Mais ce présupposé est plus que douteux. K. Dziatzko, «Joh. Tzetzes und das Plautusscholion über die alexandrinischen Bibliotheken", RhM 46, 1891, 349-70, 351

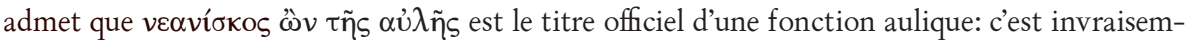

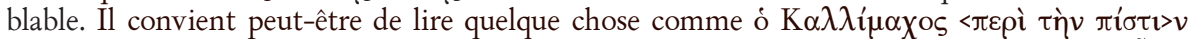

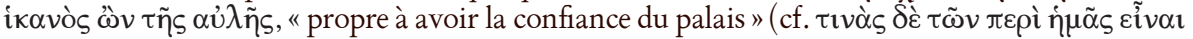

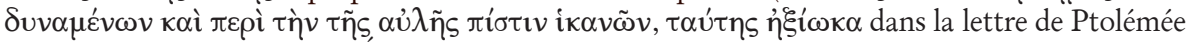
Philadelphe au grand-prêtre Éléazar chez Flavius Josèphe ant. iud. 12.47, avec la restitution de S.A. Naber, Flavii Iosephi opera omnia, Leipzig 1892, III, XII, améliorant une idée excellente de W. Dindorf, Flavii Josephi opera, Paris 1845, I, 438; le texte-source, Lettre d'Aristée à Philocrate, 37 p. 13 Mendelssohn-Wendland, est un peu différent). Une autre possibilité serait

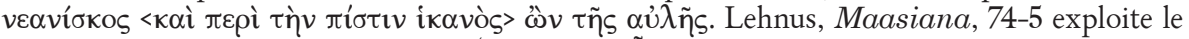

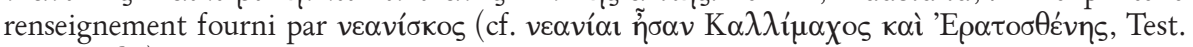
14b Pfeiffer). 
Un principe d'économie peut-être abusif a conduit certains érudits, en particulier - avec une assurance très grande - Syme 1978, 73, à faire un seul homme du Macer ami d'Ovide, du Pompeius fils de Théophane de Mitylène (ami de Pompée et historien de ses res gestae) et procurateur d'Asie, du Pompeius Macer poète tragique dont il nous reste six vers ${ }^{41}$ dits par Médée, dans une tragédie homonyme (fr. 180 Snell-Kannicht ${ }^{42}$ ), et de l'auteur de deux épigrammes de l'Anthologie Palatine. Les objections plus ou moins fortes que formule White, "Pompeius Macer", ont instillé le doute chez certains sans parvenir à faire renoncer d'autres érudits ${ }^{43}$ aux anciennes identifications. Il vaut peut-être la peine de signaler qu'Ehwald, Bursians Jahresbericht 80 1894, 12 envisage de voir dans le fragment de la Médée de Macer... une traduction de la Médée d'Ovide ! Ce dernier parle de sa tragédie à son ami Macer en $a m .2 \cdot 18.13-18^{44}$.

Merkel, P. Ovidii Nasonis Tristium, 217 allègue en faveur de son identification du destinataire de 3.14 les vers 43-44, saepe aliquod quaero uerbum nomenque locumque, Nec quisquam est a quo certior esse queam, qui évoqueraient très bien un grammaticus ${ }^{45}$. À la suite de Peter, Historicorum Romanorum reliquiae, Volumen alterum, CIII, on peut faire valoir que l'incipit, Cultor et antistes doctorum sancte uirorum, serait une bonne périphrase pour désigner un bibliothécaire : comparer, à propos de la Bibliothèque

${ }^{41}$ Leur beauté frappe F.G. Welcker, Die griechischen Tragödien mit Rücksicht auf den epischen Cyclus, Dritte Abtheilung, Bonn 1841, 1330, qui attribue les vers à une Médée. Il considère le tragique comme ne faisant qu'un avec l'homme chargé par Auguste d'" organiser les bibliothèques » et refuse le changement, chez le citateur du fragment, Stobée, de Macer en Marcus, changement qu'il croit dû à l'intention de substituer un Grec à un Romain, sous la plume de qui Welcker lui-même trouve ces vers inattendus. Welcker ne songe pas que le tragique serait d'origine grecque s'il était identifié au procurateur d'Asie fils de Théophane de Mitylène. "Those who conflate the procurator with the tragedian quoted by Stobaeus must believe that Pompeius Macer was ambidextrous, composing verse in major genres with equal facility in Greek and Latin ", écrit White, "Pompeius Macer', 216 n. 27. Cette remarque est étrange: la peritia utriusque sermonis surprendrait-elle chez les Grecs romanisés ou les Romains hellénisés autant qu'elle est inhabituelle chez les antiquisants d'aujourd'hui?

${ }^{42}$ B. Snell, Tragicorum Graecorum fragmenta. Editio correctior et addendis aucta, curavit R. Kannicht, Göttingen 1986, 312-3 rassemble les témoignages relatifs à cette " figure de synthèse ". Il suggère de rapporter les deux épigrammes au fils de son $\mathrm{Cn}$. Pompeius Macer (cf. F. Jacobs, Animadversiones in epigrammata Anthologiae Graecae (III 3), Leipzig 1814, 941-2; A.S.F. Gow, D.L. Page, The Garland of Philip, Cambridge 1968, II, 468). Voir aussi, avec la bibliographie qu'ils indiquent, G.O. Hutchinson, Greek to Latin, Frameworks and Contexts for Intertextuality, Oxford 2013, 144 et Houston, Inside Roman Libraries, 220 n. 13.

${ }^{43}$ Ainsi K. Wachtel dans PIR ${ }^{2}$ P 625 (1998) et Bowie, «Libraries», 245-6.

44 J.C. McKeown, Ovid: Amores, Volume III, A Commentary on Book Two, Leeds 1998, 383 et 393-4, qui récuse l'identification de l'ami d'Ovide avec le fils (ou le petit-fils) de Théophane de Mitylène, ignore les vers de Pompeius Macer le tragique.

${ }^{45}$ C'est aussi l'avis de Bunte, De C. Julii Hygini, 9. 
Palatine, 3.1.63-64 quaeque uiri docto ueteres peperere (cepere la plupart des mss., corr. R. Ellis, suivi par Hall, Ovidius) nouique Pectore lecturis inspicienda patent. Je suggère de voir dans l'initiale du premier mot, C(ultor), une allusion à l'initiale du prénom d'Hygin $C$ (aius). Que si l'on trouve cette idée bien extraordinaire et sans analogie, j’invoque « le double acrostiche décelé par J. Schwartz dans les lettres initiales des deux premiers vers des pièces 3,4 , 6, 7 et 8 du livre 2 [des Pontiques]: MACER NATAQ(ue)»(Irigoin, Le poète grec au travail, 487 et 491, auquel je renvoie pour le détail). Est-il exagéré de considérer que le poète qui fait un tel acrostiche peut très bien utiliser l'initiale d'un poème pour faire allusion au prénom du destinataire " caché » d'une lettre poétique ? Je propose également de rapporter palam, au v. 18, cetera fac curae sit tibi turba palam, à la bibliothèque publique en rapprochant lecturis inspicienda patent. Les adeptes des échos sonores ${ }^{46}$ n'hésiteront peut-être pas à voir aussi dans palam une allusion à Palatium, Palatinum, mais c'est une hypothèse hardie ${ }^{47}$. Toutefois on ne saurait lui opposer la différence de

\footnotetext{
${ }^{46}$ Sur ces échos et anagrammes, fréquents dans la poésie ancienne, voir P. Mitsis, I. Ziogas, edd., Wordplay and Powerplay in Latin Poetry, Berlin/Boston 2016, passim et notamment l'index (452) s. u. "wordplay ", Liberman, "L'elogio pindarico», 151 et G. Liberman, édition commentée de la monobiblos (Cynthia) de Properce, à paraître (2019), à Properce 1.22.2 quaeris PRO nostra semPER amiciTIA (allusion anagrammatique à la gens Propertia). Dans un billet (Suétone de poetis p. 47 Reifferscheid; écrits et dits d'Auguste p. 160 Weichert, 23 Malcovati ${ }^{5}, 248$ Biasi-Ferrero) adressé à Horace et dont j'établis le texte à la fin de cette note, Auguste paraît se moquer du Vinnius Asina qui lui apporta un recueil d'Horace (cf. epist. 1.13) en l'appelant Onysius, par une triple allusion à son cognomen (övos), à son nom gentilice (Onysius évoque Dionysius et Dionysos, et fait par ce truchement allusion à uinum dans Vinnius, cf. Horace epist. 1.13.14) et peut-être (Onysius / onustus) au poids léger de l'ouvrage (thème de plaisanterie chez Auguste et dans l'épittre d'Horace) et à la surcharge pondérale d'Horace, prise pour cible par le prince. J. Bernays, Gesammelte Abhandlungen (éd. H. Usener), II, Berlin 1885, 304-6 (paru en 1862) fut le premier à identifier le porteur de ce nom (Usener chez Bernays remarque que l'épigraphie a mis au jour Onysianus, nom d'un affranchi, cf. A. Lozano, Die griechischen Personennamen auf der iberischen Halbinsel, Heidelberg 1998, 149). La note, hélas influente, où E. Fraenkel, Horace, Oxford 1957, 20 récuse cette identification, est très mal inspirée: l'objection selon laquelle Auguste savait trop le grec pour rattacher Onysius à övos est tout à fait futile, car le jeu de mots n'a aucune prétention étymologique. Le prince, maniaque de la cryptographie (A. Weichert, Imperatoris Caesaris Augusti scriptorum reliquiae, Grimma 1846, 146-50), n’a donc pas dédaigné la cryptologie ludibunda. Voilà ce qu'il écrivit à Horace: Pertulit ad me Onysius libellum tuum, quem ego, ut accuso te, ita (accuso te ita Lib. : accusantem mss. : ut accusem te Bentley), quantuluscumque est, boni consulo. Vereri autem mihi uideris ne maiores libelli tui sint quam ipse es. Sed si (si $\mathrm{B}$ : omis dans les autres mss.) tibi statura deest, corpusculum non deest; itaque licebit in

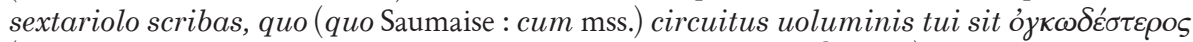
(F. Leo chez Fraenkel : ó $\kappa \kappa \omega \delta \varepsilon ́ \sigma \tau \alpha \tau o \varsigma$ mss. avec des graphies différentes), sicut est uentriculi tui. Auguste invite Horace, s'il veut que ses libelli ne soient pas plus grands que lui-même, à préférer, comme support de l'écriture, au rouleau de papyrus long et étroit un vase court et ventru comme lui (voir T. Birt, Die Buchrolle in der Kunst, Leipzig 1907, 39).

${ }^{47}$ Toutefois cette hardiesse n'est rien en regard de celle dont W. Bellamy, Shakespeare's Verbal Art, Newcastle upon Tyne, 2015, 215-17 fait montre dans ses libres interprétations anagrammatiques d'Ovide ars amatoria 2.113-26 (entre autres).
} 
quantité entre la syllabe finale de palam et la seconde syllabe de Palatium, Palatinum, car Grecs et Romains sont loin de tenir toujours compte de la quantité dans leurs jeux de mots: comparer Propertius avec première syllabe brève et pro monosyllabe long dans l'anagramme de Properce 1.22.248; D. Konstan, «A Pun in Virgil's Aeneid (4.492-93)?», CPh 95, 2000, 75 avec bibliographie; G. Liberman, Commentaire critique des Silves de Stace, Paris 2010, 169 à Stace silv. 2.1.95. Il serait aussi hardi d'arguer qu'Horace oppose priuatas et Palatinus dans le passage (epist. 1.3.15-17) où il laisse entendre que Celsus Albinovanus ${ }^{49}$ tend à préférer à l'originalité et au fonds personnels, priuatas opes, le pillage des livres de la Palatine : quid mihi Celsus agit, monitus multumque monendus Priuatas ut quaerat opes et tanger ${ }^{50}$ uitet Scripta Palatinus quaecumque recepit Apollo. Il n'est rien moins qu'impossible que, pris dans un autre sens que celui de " gardien $»^{51}$, antistes sancte présente le destinataire de l'élégie comme prêtre du temple d'Apollon Palatin: rapprocher custos... praepositus... sancto loco (3.1.67-8) à propos du responsable des bibliothecae (templi) Apollinis ${ }^{52}$. Le cognomen d'origine grecque Hyginus - nom d'un docteur chez Martial 10.56.4 ${ }^{53}$ - signifie en latin saluber, salutaris. Or Ovide développe une métaphore médicale, destinée à éclairer le lecteur suffisamment attentif : en jouant sur le mot corpus, corps physique et corpus textuel, il demande à son correspondant de retenir à Rome son corpus, retine corpus in Vrbe meum (8). Ce corpus, le poète le présente comme mutilé et c'est au " docteur " Hygin qu'il demande de contribuer à rétablir son intégrité partiellement (car une restauration complète est impossible, vu la condamnation qui pèse sur l'Art d'aimer) : ecquid, ut incolumem quondam celebrare solebas, Nunc quoque, ne uidear totus abesse, caues?

${ }^{48}$ Voir n. 46.

${ }^{49}$ Voir Syme, History in Ovid, 90.

${ }^{50}$ Tangere, "violer ", c'est-à-dire ne pas respecter l'inviolabilité de ce qui est sous la protection d'Apollon (A. Kiessling, R. Heinze Q. Horatius Flaccus, Briefe erklärt von A. Kiessling, Bearbeitet von R. Heinze, Berlin 1914, 40b), " mild gesagt für compilare " (L. Müller, Satiren und Epistel des Horaz mit Anmerkungen von Lucian Müller, II. Teil: Episteln, Prague/Vienne/Leipzig, 1893, 39).

${ }^{51}$ Voir Owen, Tristia, 71 et, pour le sens de " prêtre ", met. 13.410 antistita Phoebi et 632633 antistite Phoebus rite colebatur, pont. 4.8.25. Apollon Palatin s'appelle Phoebus dans fast. 4.951-954 et met. 15.865 .

${ }^{52}$ Langie, Les bibliothèques publiques dans l'ancienne Rome et dans l'Empire Romain, 144 imagine que, Hygin s'étant retiré, le custos est un prêtre ou un quindécemvir. Je ne crois pas à cette hypothèse, mais elle a le mérite de mettre en relief l'aspect sacré que confère aux bibliothèques leur inclusion dans le temple d'A pollon (sur cette inclusion, voir Gardthausen, Augustus, I, 965-6 et II, 579-81 et Corbier, "De la maison», 898-912 = Corbier, Donner à voir, 173-9). Carcopino, Rencontres, 145-7 s'égare tout à fait en voyant dans la façon dont Ovide s'adresse à son correspondant la preuve d'« une communauté mystique » entre pythagorisants.

${ }^{53}$ On trouve chez Galien úyıııvó substantif au sens de "spécialiste des questions de santé ", voir K. Luchner, Philiatroi. Studien zum Thema der Krankheit in der griechischen Literatur der Kaiserzeit, Göttingen 2004, 55. 
(3-4). On peut rattacher au vocabulaire médical nocuere (6), relatif au mal que causèrent à Ovide les trois livres de l'Art d'Aimer, et aussi contagia (17), dans un passage concernant cette même œuvre : tres mihi sunt nati contagia nostra secuti. Tel est le texte transmis. On explique que le mal dont souffrit Ovide s'est étendu par contagion à l'Art d'aimer, mais le tour contagia nostra sequi au sens d'" être contaminé par moi " (" that have contracted pollution from me ", Owen, Tristia, et pareillement Luck, Tristia) a légitimement éveillé le soupçon: Hall, Ovidius, lit ingénieusement tres mihi sunt nati, contagia nostra, secandi, " il convient de me retrancher trois de mes fils, contaminés par moi $\star^{54}$. J'objecte que les trois livres de l'Art d'aimer, condamnés par le prince, ont déjà été retranchés du corpus mutilé du poète, comme semblent le montrer les v. 5-6, suscipis exceptis ecquid mea carmina solis Artibus artifici quae nocuere suo? (cf. 3.1.65-6 et, aussi, à propos de la bibliothèque privée d'Ovide à Rome, 1.1.111-2 ${ }^{55}$ ainsi que, relativement à la bibliothèque privée de son ami Brutus ${ }^{56}$, Pont. 1.1.12). Je suggère donc $<$ re $>$ secti, mot très bien représenté chez Ovide et tout à fait propre à continuer la métaphore mise en œuvre par le poète : "trois de mes fils, contaminés par moi, m'ont été retirés (comme par exérèse) ». Les liens unissant à la médecine Apollon ${ }^{57}$ - le père d'Esculape, qui remit sur pied Hippolyte dilacéré58 - donnent de la pertinence au fait que le « docteur " Hygin peut aussi, si je vois juste, être considéré comme l'antistes sanctus du dieu: il fallait au moins cela pour être jugé capable de restituer au " corps " mutilé du poète une partie de son intégrité. La figure littéraire de ce grammairien " médecin » prêtre d'Apollon évoque Nicandre de Colophon, poète, certes, et l'une des source des Métamorphoses

${ }^{54}$ E. Baeza Angulo, Publio Ovidio Nasón, Tristezas, Madrid 2005, revient au texte transmis: je ne peux voir là un progrès. Hall transcrit deux conjectures, necandi (J.L. Richmond) et tegendi (W.S. Watt, dont Hall indique aussi la conjecture conuicia pour contagia), qui ont une valeur diagnostique.

${ }^{55}$ Voir Merkel, P. Ovidii Nasonis Tristium, 15: ueretur igitur poeta ut in bibliothecis extent libri artis amatoriae neue iussu Principis inde remoti sint, ainsi que $\mathrm{R}$. Ehwald, Ad historiam carminum Ovidianorum recensionemque symbolae. II., progr. Gotha 1892, 9 et T. Keith Dix, «Ovid strikes out: Tristia 3.1 and the First Public Libraries at Rome», AugAge 8, 1988, 27-35.

56 Voir Syme, History in Ovid, 80.

${ }^{57}$ Célébration d'Apollon Palatin comme médecin dans le Carmen Saeculare d'Horace, 63-4 (cf. J.F. Miller, Apollo, Augustus and the Poets, Cambridge 2009, 178). J. Gagé, Apollon romain, Paris 1955, consacre la première partie de son livre à Apollo Medicus. F.G. Welcker, Griechische Götterlehre, Göttingen 1860, II, 373 mentionne une mystérieuse attestation épigraphique d'Apollon Hygieus - confusion avec Agyieus ? Aux v. 632-3 de met. 15, salutifera... sorte pour salutifera... arte (à propos d'Apollon delphique, que les Romains voudraient voir anéantir un empoisonnement de l'air dont ils sont les victimes) est, je crois, une faute, qui paraît avoir jusqu’à maintenant échappé; comparer salutari... arte dans le passage mentionné du carmen horatien.

${ }^{58}$ Ovide fast. 6.733-62, met. 15.497-546. Cedent arti tristia fata meae, dit Esculape à Hippolyte (fast. 6.748). 
d'Ovide, mais aussi grammairien titulaire d'un sacerdoce héréditaire d'Apollon Clarien et, d'après un renseignement contesté de la Souda, médecin ${ }^{59}$.

L'avant-dernier distique, où Ovide dit craindre que son latin ne soit devenu barbare, tire son sel du fait qu'il s'adresse à un grammairien : crede mihi, timeo ne sint inmixta Latinis Inque meis scriptis Pontica uerba legas. Housman ${ }^{60}$ a montré en 1916 que ce texte était bancal — on n'a étonnamment pas encore, à ma connaissance, tenté de le défendre en expliquant que la claudication dont il est affligé vise à rendre sensible la barbarie qu'Ovide redoute pour son latin. Housman a presque réussi à imposer son ingénieuse correction timeo ne Sintia (= Sintica) mixta Latinis, qui introduit chez Ovide la mention d'un peuple des bords du Strymon, les Sintes ${ }^{61}$. Mais cette correction doit, à mon avis, être écartée, parce que Sintia et Pontica uerba constitue une répétition gênante de la même idée, Sintius et Ponticus désignant la même chose, la langue Gète, selon un usage poétique et ovidien abondamment illustré par Housman lui-même à propos de ce passage. Hall, Ovidius, ${ }^{62}$ préfère sa propre conjecture timeo ne sint fera mixta Latinis, qui échappe à l'objection formulée contre Sintia mais se heurte à une autre objection : $L a$ tinis appelle, pour faire contraste avec lui, un adjectif substantivé du même type que lui, c'est-à-dire tel que Sintia. Je suggère qu'Ovide avait écrit timeo ne Pontica mixta Latinis inque meis scriptis barbara uerba legas, c'està-dire, selon une figure aussi illustrée par Housman, timeo ne in meis scriptis Pontica mixta Latinis barbaraque uerba legas. La chute de barbara devant uerba a peut-être entraîné un remaniement consistant à transférer Pontica devant uerba et à ajouter sint in (mixta) pour faire le vers et (en apparence) simplifier la construction. Comparer pont. 4.13.19-20 a, pudet: et Getico scripsi sermone libellum Structaque sunt nostris barbara uerba modis. Il me semble que la restitution de barbara accroit le sel du passage: personne n'était plus sensible aux barbara uerba que le grammairien et vétilleux commentateur de Virgile ${ }^{63}$ Hygin, et à personne mieux qu’à lui Ovide

${ }^{59}$ Données commodément rassemblées chez O. Schneider, Nicandrea, Leipzig 1856, XVIIXIX. Le tome I où feu J.-M. Jacques devait aborder la biographie de Nicandre et qui devait couronner le monument que constituent les tomes II (2002) et III (2007) de son édition n'est pas paru.

${ }^{60}$ A.E. Housman, The Classical Papers of A. E. H., Cambridge 1972, 3, 923-4.

${ }^{61}$ Housman approuve la correction Sinti nec militis (nec Sinti militis E.J. Kenney) dans trist. 4.1.21 mais il ne mentionne pas la conjecture Sintica terra (R. Ellis, "Owens' Tristia», Hermathena, 7, 1890, 192) en 4.4b.9.

${ }^{62}$ Baeza Angulo, Tristezas, revient à la conjecture de Housman.

${ }^{63}$ L'érudit puriste Hygin trouve chez Virgile des défauts d'expression, des impropriétés et, sur le fond, des inexactitudes, des anachronismes, des contradictions. Voir, avec des jugements divers sur Hygin critique littéraire ou textuel, Ribbeck, Prolegomena, 117-21 (sani iudicii hominem sermonisque Latini optime gnarum), S. Timpanaro, Per la storia della filologia virgiliana antica, Rome 1986, 51-67 et Virgilianisti antichi e tradizione indiretta, Florence 2001, 13-23, M. Mülke, Der Autor und sein Text, Die Verfälschung des Originals im Urteil antiker Autoren, Berlin/New York 2008, 39-40 et R. Tarrant, Vergil, Aeneid Book XII, 2012 
ne pouvait adresser une requête d'indulgence, à la fois coquetterie de poète et moyen de susciter la compassion. Quoi de plus lamentable en effet qu'un poète romain surdoué à qui l'exil ferait perdre son latin?

Cambridge 2012, 121-2 à Virgile Aen. 12.120 (excellente restitution par Hygin d'un mot très rare, limus ou limum [cf. limocinctus] disparu au profit d'un mot banal et non pertinent, linum). G.B. Conte, Critical Notes on Virgil. Editing the Teubner Text of the Georgics and the Aeneid, Berlin/Boston 2016, s'amuse aux dépens de "the good old Julius Hyginus " à propos de la leçon pédante amaror ou, ce qui n'est pas mieux, sensus... amaror, pour sensu... amaro, que le préfet de la Palatine veut restituer à Virgile (georg. 2.247) en s'appuyant sur un ms. ex domo atque ex familia Vergili (comparer, pour la phraséologie, trist. 1.1.1056). Hygin ne manquerait pas de matière pour critiquer son lointain successeur italien: voir G. Liberman, CR 62, 2012, 149-51 et Annuaire de l'École pratique des hautes études, Section des sciences historiques et philologiques, 148, 2017, 105-14. 
